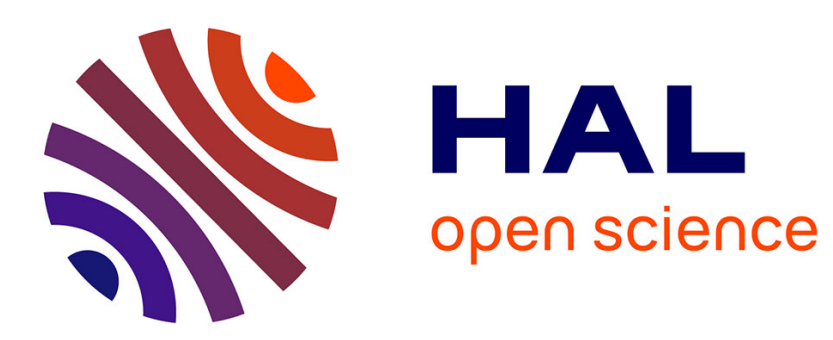

\title{
Critical phenomena near the antiferromagnetic quantum critical point of heavy fermions
}

\author{
M. Lavagna, Catherine Pepin
}

\section{To cite this version:}

M. Lavagna, Catherine Pepin. Critical phenomena near the antiferromagnetic quantum critical point of heavy fermions. Physical Review B: Condensed Matter and Materials Physics (1998-2015), 2000. hal-01896145

\section{HAL Id: hal-01896145 \\ https://hal.science/hal-01896145}

Submitted on 16 Oct 2018

HAL is a multi-disciplinary open access archive for the deposit and dissemination of scientific research documents, whether they are published or not. The documents may come from teaching and research institutions in France or abroad, or from public or private research centers.
L'archive ouverte pluridisciplinaire HAL, est destinée au dépôt et à la diffusion de documents scientifiques de niveau recherche, publiés ou non, émanant des établissements d'enseignement et de recherche français ou étrangers, des laboratoires publics ou privés. 


\title{
Critical phenomena near the antiferromagnetic quantum critical point of heavy fermions
}

\author{
M. Lavagna ${ }^{1, *}$ and C. Pépin ${ }^{2}$ \\ ${ }^{1}$ Commissariat à l'Energie Atomique, DRFMC /SPSMS, 17, rue des Martyrs, 38054 Grenoble Cedex 9, France \\ ${ }^{2}$ Department of Physics, University of Oxford, 1 Keble Road, Oxford OX1 3NP, United Kingdom
}

(Received 18 January 2000; revised manuscript received 27 April 2000)

\begin{abstract}
We present a paper of the critical phenomena around the quantum critical point in heavy-fermion systems. In the framework of the $S=1 / 2$ Kondo lattice model, we introduce an extended decoupling scheme of the Kondo interaction, which allows one to treat the spin fluctuations and the Kondo effect on an equal footing. The calculations, developed in a self-consistent one-loop approximation, lead to the formation of a damped collective mode with a dynamic exponent $z=2$ in the case of an antiferromagnetic instability. The system displays a quantum-classical crossover at finite temperature depending on how the energy of the mode, on the scale of the magnetic correlation length, compares to $k_{B} T$. The low-temperature behavior, in the different regimes separated by the crossover temperatures, is then discussed for both two- and three-dimensional systems.
\end{abstract}

\section{INTRODUCTION}

A central issue in the thermal properties of heavy-fermion compounds is the discovery of a non-Fermi liquid (NFL) behavior in systems close to an antiferromagnetic quantum phase transition. ${ }^{1}$ Such a behavior has been discovered in a series of compounds containing cerium or uranium, for example, $\mathrm{CeCu}_{6-x} \mathrm{R}_{x}(\mathrm{R}=\mathrm{Au}, \mathrm{Ag}),{ }^{2} \mathrm{CeIn}_{3}, \mathrm{CePd}_{2} \mathrm{Si}_{2},{ }^{3}$ $\mathrm{CeNi}_{2} \mathrm{Ge}_{2},{ }^{4} \quad \mathrm{U}_{1-x} \mathrm{Y}_{x} \mathrm{Pd}_{3},{ }^{5}$ and $\mathrm{Ce}_{x} \mathrm{La}_{1-x} \mathrm{Ru}_{2} \mathrm{Si}_{2} .{ }^{6} \quad$ In $\mathrm{CeCu}_{5.9} \mathrm{Au}_{0.1}$, the specific heat $C$ depends on $T$ as $C / T$ $\sim-\ln \left(T / T_{0}\right)$, the magnetic susceptibility as $\chi \sim 1-\alpha \sqrt{T}$, and the $T$-dependent part of the resistivity as $\Delta \rho \sim T$ (instead of $C / T \sim \chi \sim$ const and $\Delta \rho \sim T^{2}$ in the Fermi-liquid state). Most interestingly, the breakdown of the Fermi-liquid behavior can be tuned by alloying (chemical pressure) or by applying a hydrostatic pressure or a magnetic field. The origin of this NFL behavior is a problem of current debate.

Different scenarios have been proposed in order to elucidate the NFL behavior at the quantum critical point (QCP). On the one hand, there are scenarios involving strong disorder to account for the non-Fermi liquid properties, including (i) a distribution of the Kondo temperature $P\left(T_{K}\right)$ (Ref. 7) induced by disorder following a distribution of the local density-of-states, and (ii) the possibility of the formation of a Griffiths phase at the quantum critical point. ${ }^{8,9}$ On the other hand, starting from a clean or rather weakly disordered system, there is a scenario (iii) referring to the proximity of a quantum critical point in a theory of itinerant antiferromagnetism, ${ }^{10-17}$ in which the Fermi surface is coupled to critical collective antiferromagnetic excitations. An alternative possibility has been recently put forward ${ }^{18}$ on the basis of an analysis of the neutron scattering measurements in $\mathrm{CeCu}_{5.9} \mathrm{Au}_{0.1}$. The unusual spin dynamics that has been observed suggests (iv) the existence of critically screened local magnetic excitations due to the formation of local moments. Finally, (v) a variant of the quadrupolar Kondo effect ${ }^{19}$ in which the interactions of the rare-earth (actinide) internal degrees of freedom with the conduction electrons are reduced to a single impurity multichannel
Kondo effect has been proposed. As it stands, the situation remains highly controversial.

In this paper, we adopt the point of view (iii) of the proximity to a quantum critical point. The effect of a nonzero temperature on quantum critical points is a long-standing problem encountered in itinerant magnetism. We refer to the papers of Hertz ${ }^{20}$ and Millis ${ }^{11}$ using renormalization-group techniques, and of Moriya ${ }^{21}$ introducing a self-consistent renormalization (SCR) theory of spin fluctuations. These theories yield a rich phase diagram with, in addition to the ordering temperature $T_{N}$, a series of crossover temperatures separating different regimes of behaviors. They are well adapted for the treatment of spin fluctuations as present in the single-band Hubbard model but essentially do not account for the Kondo effect.

Indeed, the model that is believed to describe the physics of heavy fermions is the Kondo lattice model ${ }^{22}$ derived from the periodic Anderson model in a given limit. The large $N$ expansions, ${ }^{23}$ which have been carried out for these models (where $N$ represents simultaneously the degeneracy of the conduction electrons and of the spin channels) give a good description of the Kondo effect. But, unlike the abovementioned spin-fluctuation theories, they fail to account for the spin fluctuations since the Ruderman-Kittel-KasuyaYosida interactions only appear at the order $1 / N^{2}{ }^{24}$

Hence our motivation to set up an approach of the $S$ $=1 / 2$ Kondo lattice model $(N=2)$ that enlarges the standard $1 / N$ expansion theories up on the spin-fluctuation effects. Our previous paper ${ }^{25}$ presented such an approach by performing a generalized Hubbard-Stratonovich transformation on the Kondo interaction term of the Hamiltonian, which makes three types of fields (magnetization densities and Kondo type) appear on an equal footing. The dynamical spin susceptibility was derived in a one-loop expansion associated with the Gaussian fluctuations of the magnetization density fields around their saddle-point values.

With the aim to describe the critical phenomena around the quantum critical point of the heavy-fermion systems, we propose to push the treatment of the $S=1 / 2$ Kondo lattice model in a self-consistent one-loop approximation in com- 
plete analogy with the SCR theory of spin fluctuations developed for the Hubbard model. ${ }^{21,26,27}$ As in itinerant magnetism, we will show how the system displays a quantumclassical crossover at finite temperature depending whether the temperature is lower or higher than the characteristic energy scale of the collective mode associated with the magnetic instability. The low-temperature behavior in the different regimes essentially depends on the value of the dynamic exponent $z$ of the mode and on the dimensionality $d$ of the problem. In the case of an antiferromagnetic instability, $z$ is found to be equal to 2. Heavy-fermion systems are usually believed to be three dimensional. However, a recent proposal ${ }^{28}$ based on the neutron-scattering data obtained in $\mathrm{CeCu}_{6-x} \mathrm{Au}_{x}$, stipulates that the critical magnetic fluctuations, which the quasiparticles are coupled to, are simply two dimensional. So both situations $d=2$ and $d=3$ will be considered.

The rest of the paper is organized as follows. In Sec. II, we introduce the self-consistent one-loop approximation method for the Kondo lattice model using a generalized Hubbard-Stratonovich transformation on the Kondo interaction term. The approach combines both aspects of the $1 / N$ expansion and of the self-consistent renormalization theory of spin fluctuations. The calculations, presented in the functional integral formalism, closely follow the presentation made by Hertz and Klenin ${ }^{27}$ for the Hubbard model. Hence, we extract the general expression of the dynamical spin susceptibility extending the results of the previous paper ${ }^{25}$ to a self-consistent treatment. Section III gives a discussion on the nature of the damped collective mode associated to the proximity of the magnetic transition with a dynamic exponent $z=2$ in the antiferromagnetic case. In Sec. IV, we derive the resulting quantum-classical crossover. This occurs at a finite temperature $T$, which depends on how the energy of the mode, on the scale of the magnetic correlation length $\xi$ compares to $k_{B} T$. The low-temperature behavior of the system in the different regimes, separated by the crossover temperatures, is then discussed in Sec. V. Concluding remarks follow in Sec. VI.

\section{SELF-CONSISTENT ONE-LOOP APPROXIMATION TO THE KONDO LATTICE MODEL}

This section extends the approach introduced in our previous paper $^{25}$ to a self-consistent one-loop approximation. Our starting point is a path-integral treatment of the Kondo lattice model Hamiltonian

$$
\begin{aligned}
H= & \sum_{k \sigma} \varepsilon_{k} c_{k \sigma}^{\dagger} c_{k \sigma}+J \sum_{i} \mathbf{S}_{f i} \cdot \sum_{\sigma \sigma^{\prime}} c_{i \sigma}^{\dagger} \boldsymbol{\tau}_{\sigma \sigma^{\prime}} c_{i \sigma^{\prime}} \\
& -\mu\left(\sum_{i \sigma} c_{i \sigma}^{\dagger} c_{i \sigma}-n_{c} N_{S}\right),
\end{aligned}
$$

where $\mathbf{S}_{f i}$ represents the spin $(S=1 / 2)$ of the impurities distributed on the sites (in number $N_{S}$ ) of a periodic lattice; $c_{k \sigma}^{\dagger}$ is the creation operator of the conduction electron of momentum $\mathbf{k}$ and spin quantum number $\sigma$ characterized by the energy $\epsilon_{k}=-\Sigma_{\langle i, j\rangle} t_{i j} \exp \left(i \mathbf{k} \cdot \mathbf{R}_{i j}\right) ; \boldsymbol{\tau}$ are the Pauli matrices $\left(\boldsymbol{\tau}^{x}, \boldsymbol{\tau}^{y}, \boldsymbol{\tau}^{z}\right)$ and $\boldsymbol{\tau}^{0}$ the unit matrix; $\mathrm{J}$ is the antiferromagnetic Kondo interaction $(J>0)$. The Hamiltonian is written in the grand canonical ensemble with the introduction of a chemical potential $\mu$ in order to guarantee an average number of conduction electrons per site $n_{c}<1$. The spin of the impurities is treated within the Abrikosov pseudofermion representation $\mathbf{S}_{f i}=\Sigma_{\sigma \sigma^{\prime}} f_{i \sigma}^{\dagger} \boldsymbol{\tau}_{\sigma \sigma^{\prime}} f_{i \sigma^{\prime}}$ where the local constraint $Q_{i}$ $=\Sigma_{\sigma} f_{i \sigma}^{+} f_{i \sigma}-1=0$ is implemented in the usual way, by introducing a time-independent Lagrange multiplier $\lambda_{i}$. The local Kondo interaction is decoupled using a HubbardStratonovich transformation, which introduces four auxiliary fields $\Phi_{i}(\tau), \Phi_{i}^{*}(\tau)$, and $\boldsymbol{\xi}_{f_{i}}(\tau), \boldsymbol{\xi}_{c_{i}}(\tau)$. We are then left with the effective action

$$
S_{e f f}=\int_{0}^{\beta} d \tau\left[\mathcal{L}_{0}(\tau)+H_{J}(\tau)\right]
$$

where $\mathcal{L}_{0}(\tau)$ is the Lagrangian in the absence of the interaction $\mathbf{J}$ and

$$
\begin{aligned}
H_{J}(\tau)= & \sum_{i \sigma \sigma^{\prime}}\left[c_{i \sigma}^{\dagger}(\tau) f_{i \sigma}^{\dagger}(\tau)\right] \\
& \times\left(\begin{array}{ll}
-J_{S} i \xi_{f_{i}}(\tau) \cdot \boldsymbol{\tau}_{\sigma \sigma^{\prime}} & J_{C} \Phi_{i}^{*}(\tau) \tau_{\sigma \sigma^{\prime}}^{0} \\
J_{C} \Phi_{i}(\tau) \tau_{\sigma \sigma^{\prime}}^{0} & -J_{S} i \boldsymbol{\xi}_{c_{i}}(\tau) \cdot \boldsymbol{\tau}_{\sigma \sigma^{\prime}}
\end{array}\right) \\
& \times\left(\begin{array}{l}
c_{i \sigma^{\prime}}(\tau) \\
f_{i \sigma^{\prime}}(\tau)
\end{array}\right)+J_{C} \sum_{i} \Phi_{i}^{*}(\tau) \Phi_{i}(\tau) \\
& +J_{S} \sum_{i} \boldsymbol{\xi}_{f_{i}}(\tau) \cdot \boldsymbol{\xi}_{c_{i}}(\tau)
\end{aligned}
$$

with $J_{S}=J / 4$ and $J_{C}=J / 3$. Details on the saddle-point solution and the Gaussian fluctuations can be found in our previous paper. ${ }^{25}$ The saddle-point solution transposes to $N=2$ the large- $N$ results obtained within the slave-boson meanfield theories. It gives rise to the formation of a AbrikosovSuhl resonance pinned at the Fermi level and split by a hybridization gap defining two bands $\alpha$ and $\beta$. The one-loop approximation is then obtained by considering the Gaussian fluctuations of the bosonic fields around their mean-field values. As in the conventional approaches to critical phenomena, the approach consists to integrate out the fermion fields and thereby reduce the problem to the study of an effective bosonic theory describing fluctuations of the ordering fields. But contrary to Hertz ${ }^{20}$ and Millis, ${ }^{11}$ who considered a single field corresponding to the magnetization density of one type of electron, the approach, starting from a different microscopic model, extends the description to the case of several fields, i.e., the Kondo fields on the one hand, and the $f$ - and $c$ - magnetization densities on the other hand.

We now propose to include the self-consistent effects in the one-loop approximation. We do it in a variational way and replace the full expression $S_{\text {eff }}-S_{\text {eff }}^{(0)}$ (where $S_{\text {eff }}^{(0)}$ and $F^{(0)}$ are the zeroth order effective action and the related free energy) by a trial quadratic form $\Delta \widetilde{S}_{e f f}^{(2)}=\widetilde{S}_{\text {eff }}^{(2)}-S_{\text {eff }}^{(0)}$ in the fields $\delta r_{q, \nu}, \delta \lambda_{q, \nu}, \delta \boldsymbol{\xi}_{f_{q, \nu}}$, and $\delta \boldsymbol{\xi}_{c, \nu}$ working in the radial gauge for the Kondo parameters ${ }^{29}$ using Fourier transform notations 


$$
\begin{aligned}
\Delta \widetilde{S}_{e f f}^{(2)}= & \frac{1}{\beta} \sum_{\mathbf{q}, i \omega_{\nu}}\left[\left(\delta r_{q, \nu} \delta \lambda_{q, \nu}\right) \widetilde{\mathbf{D}}_{C}^{-1}\left(\mathbf{q}, i \omega_{\nu}\right)\left(\begin{array}{c}
\delta r_{-q,-\nu} \\
\delta \lambda_{-q,-\nu}
\end{array}\right)\right. \\
& +\left(\delta \xi_{f q, \nu}^{z} \delta \xi_{c q, \nu}^{z}\right) \widetilde{\mathbf{D}}_{S}^{\|-1}\left(\mathbf{q}, i \omega_{\nu}\right)\left(\begin{array}{c}
\delta \$ x i_{f-q,-\nu}^{z} \\
\delta \xi_{c-q,-\nu}^{z}
\end{array}\right) \\
& +\left(\delta \xi_{f q, \nu}^{+} \delta \xi_{c q, \nu}^{+}\right) \widetilde{\mathbf{D}}_{S}^{\perp-1}\left(\mathbf{q}, i \omega_{\nu}\right)\left(\begin{array}{c}
\delta \xi_{f-q,-\nu}^{-} \\
\delta \xi_{c-q,-\nu}^{-}
\end{array}\right) \\
& \left.+\left(\delta \xi_{f q, \nu}^{-} \delta \xi_{c q, \nu}^{-}\right) \widetilde{\mathbf{D}}_{S}^{\perp-1}\left(\mathbf{q}, i \omega_{\nu}\right)\left(\begin{array}{c}
\delta \xi_{f-q,-\nu}^{+} \\
\delta \xi_{c-q,-\nu}^{+}
\end{array}\right)\right]
\end{aligned}
$$

where the indices $q$ and $\nu$, respectively, stand for the wave vector $\mathbf{q}$ and the bosonic Matsubara frequencies $i \omega_{\nu}$ $=2 \nu i \pi / \beta$. Note that Eq. (3) is written using the radial gauge in which the phase of the fields $\Phi_{i}$ and $\Phi_{i}^{*}$ is incorporated into the Lagrange multiplier $\lambda_{i}$, which turns out to be a field. The elements of the matrices $\widetilde{\mathbf{D}}_{C}^{-1}\left(\mathbf{q}, i \omega_{\nu}\right), \widetilde{\mathbf{D}}_{S}^{\|-1}\left(\mathbf{q}, i \omega_{\nu}\right)$ and $\widetilde{\mathbf{D}}_{S}^{\perp-1}\left(\mathbf{q}, i \omega_{\nu}\right)$ are determined variationally by minimizing the free energy or, more precisely, the upper bound to the free energy according to the Feynman variational principle $F \leqslant \widetilde{F}_{e f f}^{(2)}+1 / \beta\left\langle S_{e f f}-\widetilde{S}_{e f f}^{(2)}\right\rangle$ where $\widetilde{F}_{\text {eff }}^{(2)}$ represents the free energy related to $\widetilde{S}_{e f f}^{(2)}$ and $\langle A\rangle$ means the average of the quantity $A$ over the distribution of fields $\exp \left(-\Delta \widetilde{S}_{e f f}^{(2)}\right)$, i.e.,

$$
\langle A\rangle=\frac{\int \mathcal{D} r_{i} \mathcal{D} \lambda_{i} \mathcal{D} \boldsymbol{\xi}_{f_{i}} \mathcal{D} \boldsymbol{\xi}_{c_{i}} A \exp \left(-\Delta \widetilde{S}_{\text {eff }}^{(2)}\right)}{\left.\int \mathcal{D} r_{i} \mathcal{D} \lambda_{i} \mathcal{D} \boldsymbol{\xi}_{f_{i}} \mathcal{D} \boldsymbol{\xi}_{c_{i}}\right) \exp \left(-\Delta \widetilde{S}_{\text {eff }}^{(2)}\right)} .
$$

After minimization, one can show that $\left[\mathbf{D}_{C}^{-1}\left(\mathbf{q}, i \omega_{\nu}\right)\right]_{r \lambda}$ is equal to $(1 / 2) \partial^{2} S_{e f f} /\left(\partial r_{q, \nu} \partial \lambda_{-q,-\nu}\right)$ averaged over the mentioned distribution of fields (and equivalent expressions for the other elements). Explicitly we obtain,

$$
\begin{gathered}
\widetilde{\mathbf{D}}_{C}^{-1}\left(\mathbf{q}, i \omega_{\nu}\right)=\left(\begin{array}{cc}
J_{C}\left[1-J_{C}\left(\left\langle\bar{\varphi}_{2}\left(\mathbf{q}, i \omega_{\nu}\right)\right\rangle+\left\langle\bar{\varphi}_{m}\left(\mathbf{q}, i \omega_{\nu}\right)\right\rangle\right)\right] & -J_{C}\left\langle\bar{\varphi}_{1}\left(\mathbf{q}, i \omega_{\nu}\right)\right\rangle \\
-J_{C}\left\langle\bar{\varphi}_{1}\left(\mathbf{q}, i \omega_{\nu}\right)\right\rangle & -\left\langle\bar{\varphi}_{f f}\left(\mathbf{q}, i \omega_{\nu}\right)\right\rangle
\end{array}\right), \\
\widetilde{\mathbf{D}}_{S}^{\|-1}\left(\mathbf{q}, i \omega_{\nu}\right)=\left(\begin{array}{cc}
J_{S}^{2}\left\langle\varphi_{c c}^{\|}\left(\mathbf{q}, i \omega_{\nu}\right)\right\rangle & J_{S}\left[1+J_{S}\left\langle\varphi_{f c}^{\|}\left(\mathbf{q}, i \omega_{\nu}\right)\right\rangle\right] \\
J_{S}\left[1+J_{S}\left\langle\varphi_{c f}^{\|}\left(\mathbf{q}, i \omega_{\nu}\right)\right\rangle\right] & J_{S}^{2}\left\langle\varphi_{f f}^{\|}\left(\mathbf{q}, i \omega_{\nu}\right)\right\rangle
\end{array}\right),
\end{gathered}
$$

and an equivalent expression for the transverse spin part $\widetilde{\mathbf{D}}_{S}^{\perp-1}\left(\mathbf{q}, i \omega_{\nu}\right)$. The different bubbles $\left\langle\varphi\left(\mathbf{q}, i \omega_{\nu}\right)\right\rangle$ in the latter expressions represent the susceptibilities $\varphi\left(\mathbf{q}, i \omega_{\nu}\right)$ (cf. appendix) averaged over the distribution of fields $\exp \left(-\Delta \widetilde{S}_{e f f}^{(2)}\right)$. At that point, the problem is highly complex and cannot be solved exactly. Therefore, we introduce a local and instantaneous approximation that is equivalent to averaging over Gaussian distributions of local fields $\left(r_{i}, \lambda_{i}, \boldsymbol{\xi}_{f_{i}}, \boldsymbol{\xi}_{c_{i}}\right)$ with variances $\sigma_{r}, \sigma_{\lambda}, \sigma_{f}$, and $\sigma_{c}$ defined as

$$
\sigma_{f}^{2}=3 \sum_{\mathbf{q}, i \omega_{\nu}}\left[\widetilde{\mathbf{D}}_{S}^{\|}\left(\mathbf{q}, i \omega_{\nu}\right)_{f f}\right]
$$

and similar expressions for the three other variances. Equation (6) is obtained from Eq. (3) by letting the summation over $\left(q, i \omega_{\nu}\right)$ act on the matrix elements only, hence defining the local and instantaneous fluctuations of the various degrees of freedom. One expects that at low temperatures, the fluctuations of $r_{i}, \lambda_{i}$, and $\boldsymbol{\xi}_{c_{i}}$ only bring $T^{2}$ corrections to their saddle-point values. Those corrections are negligible compared to the $T$ dependence brought by the fluctuations of $\boldsymbol{\xi}_{f_{i}}$ and will not be considered here. So we will neglect, in the following, the fluctuations of $r_{i}, \lambda_{i}$, and $\xi_{c_{i}}$, and focus on the fluctuations of $\boldsymbol{\xi}_{f_{i}}$ only. Then $\left\langle\varphi\left(\mathbf{q}, i \omega_{\nu}\right)\right\rangle$ is simply given by

$$
\left\langle\varphi\left(\mathbf{q}, i \omega_{\nu}\right)\right\rangle=\frac{\left(\int \mathcal{D} \xi_{f_{i}} \varphi\left(\mathbf{q}, i \omega_{\nu}\right) \exp \left[-\xi_{f_{i}}^{2} /\left(2 \sigma_{f}^{2}\right)\right]\right)}{\left(\int \mathcal{D} \xi_{f_{i}} \exp \left[-\xi_{f_{i}}^{2} /\left(2 \sigma_{f}^{2}\right)\right]\right)}
$$

Equations (6)-(7) are the basic equations of the paper from which the dynamical spin susceptibility is extracted and the critical phenomena around the QCP are studied. Let us note that, when the averages acting on the various bubbles $\varphi\left(\mathbf{q}, i \omega_{\nu}\right)$ in Eq. (5) are omitted, one recovers the standard results of the random-phase approximation. As pointed out by Lonzarich and Taillefer, ${ }^{30}$ the corresponding selfconsistent renormalized spin fluctuation SCR-SF procedure can be analyzed within the Ginzburg-Landau formalism in which the local free energy is expanded in terms of a small and slowly varying order parameter $\mathbf{m}(\mathbf{r})$ as $f(\mathbf{r})=f_{0}$ $+\frac{1}{2} a|\mathbf{m}(\mathbf{r})|^{2}+\frac{1}{4} b|\mathbf{m}(\mathbf{r})|^{4}+\frac{1}{2} c \sum_{i}\left|\boldsymbol{\nabla} m^{i}(\mathbf{r})\right|^{2}+O(6) \quad$ and the following approximation is made $|\mathbf{m}(\mathbf{r})|^{4}$ $=\left\langle|\mathbf{m}(\mathbf{r})|^{2}\right\rangle|\mathbf{m}(\mathbf{r})|^{2}$. Hence $\left\langle|\mathbf{m}(\mathbf{r})|^{2}\right\rangle$ is evaluated via the fluctuation-dissipation theorem.

The expression of the dynamical spin susceptibility is then derived in the usual way, by adding a source term $-2 \mathbf{S}_{f} \cdot \mathbf{B}$ to the Hamiltonian. In the Matsubara representation, one gets 


$$
\chi_{f f}\left(\mathbf{q}, i \omega_{\nu}\right)=\frac{\left\langle\varphi_{f f}\left(\mathbf{q}, i \omega_{\nu}\right)\right\rangle}{1-J_{S}^{2}\left[\left\langle\varphi_{f f}\left(\mathbf{q}, i \omega_{\nu}\right)\right\rangle\left\langle\varphi_{c c}\left(\mathbf{q}, i \omega_{\nu}\right)\right\rangle-\left\langle\varphi_{f c}\left(\mathbf{q}, i \omega_{\nu}\right)\right\rangle^{2}-\frac{2}{J_{S}}\left\langle\varphi_{f c}\left(\mathbf{q}, i \omega_{\nu}\right)\right\rangle\right]}
$$

In the low-frequency limit, one can easily check that the dynamical spin susceptibility may be written in terms of intra- and interband suceptibilities corresponding, respectively, to particle-hole pair excitations within the lower $\alpha$ band and from the lower $\alpha$ to the upper $\beta$ band

$$
\chi_{f f}\left(\mathbf{q}, i \omega_{\nu}\right)=\frac{\left\langle\bar{\chi}_{\alpha \alpha}\left(\mathbf{q}, i \omega_{\nu}\right)\right\rangle+\left\langle\bar{\chi}_{\alpha \beta}\left(\mathbf{q}, i \omega_{\nu}\right)\right\rangle}{1-J_{S}^{2}\left\langle\chi_{\alpha \alpha}\left(\mathbf{q}, i \omega_{\nu}\right)\right\rangle\left\langle\bar{\chi}_{\alpha \beta}\left(\mathbf{q}, i \omega_{\nu}\right)\right\rangle}
$$

The expressions of the susceptibilities $\chi_{\alpha \alpha}\left(\mathbf{q}, i \omega_{\nu}\right)$ and $\bar{\chi}_{\alpha \beta}\left(\mathbf{q}, i \omega_{\nu}\right)$, corresponding to intra- and interband particlehole excitations are given in the Appendix. The latter expression is reminiscent of the behavior proposed by Bernhoeft and Lonzarich ${ }^{31}$ to explain the neutron scattering observed in $U \mathrm{Pt}_{3}$ with the existence of both a "slow" and a "fast" component in $\chi^{\prime \prime}(\mathbf{q}, \omega) / \omega$ due to spin-orbit coupling. Also in a phenomenological way, the same type of feature has been suggested in the duality model developed by Kuramoto and Miyake. $^{32}$ To our knowledge, the proposed approach provides the first microscopic derivation from the Kondo lattice model of such a behavior. Expanding the various susceptibilities $\varphi$ up to the second order in $\xi_{f_{i}}$, and making use of Eq. (7), one can draw from Eq. (9) the following expression of the dynamical spin susceptibility (taking the analytical continuation $\left.i \omega_{\nu} \rightarrow \omega+i \delta\right)$

$$
\chi_{f f}(\mathbf{q}, \omega)=\frac{\chi_{\alpha \alpha}(\mathbf{q}, \omega)+\bar{\chi}_{\alpha \beta}(\mathbf{q}, \omega)}{1-J_{S}^{2} \chi_{\alpha \alpha}(\mathbf{q}, \omega) \bar{\chi}_{\alpha \beta}(\mathbf{q}, \omega)+\lambda \sigma_{f}^{2}},
$$

where $\lambda$ is a constant which can be evaluated from the expansion of $\left\langle\chi_{\alpha \alpha}\left(\mathbf{q}, i \omega_{\nu}\right)\right\rangle$ and $\left\langle\bar{\chi}_{\alpha \beta}\left(\mathbf{q}, i \omega_{\nu}\right)\right\rangle$ up to the second order in $\sigma_{f}^{2}$. The variance $\sigma_{f}^{2}$ defined by Eq. (6) can as well be expressed as a function of $\chi_{f f}\left(\mathbf{q}, i \omega_{\nu}\right)$

$$
\sigma_{f}^{2}=\sum_{\mathbf{q}, i \omega_{\nu}} \chi_{f f}\left(\mathbf{q}, i \omega_{\nu}\right)=\frac{3}{\pi} \sum_{\mathbf{q}} \int_{0}^{+\infty} \operatorname{coth} \frac{\beta \omega}{2} \chi_{f f}^{\prime \prime}(\mathbf{q}, \omega) d \omega .
$$

The two last equations [Eqs. (10)-(11)] provide a selfconsistent determination of $\sigma_{f}^{2}$ and hence of the spin susceptibility. We will successively present now (i) in Sec. III, the result for the dynamical spin susceptibility in the randomphase approximation (RPA) giving information on the nature of the collective mode that is involved; (ii) then in Sec. IV, the implications of that mode in the critical phenomena around the quantum critical point.

\section{RPA-DYNAMICAL SPIN SUSCEPTIBILITY NEAR THE ANTIFERROMAGNETIC WAVE VECTOR: DISCUSSION ON THE NATURE OF THE COLLECTIVE MODE}

As pointed out in Sec. II, the RPA corresponds to the absence of any averaging in the different equations. Thereby the expression of the dynamical spin susceptibility at the RPA level is given by Eq. (10) taking $\sigma_{f}^{2}=0$. We now discuss the $\mathbf{q}$ and $\omega$ dependence of the RPA dynamical spin susceptibility around the antiferromagnetic wave vector $\mathbf{Q}$.

The bare intraband susceptibility $\chi_{\alpha \alpha}(\mathbf{Q}+\mathbf{q}, \omega)$ is well approximated at $|\mathbf{q}| \ll|\mathbf{Q}|$ by a Lorentzian $\chi_{\alpha \alpha}(\mathbf{Q}+\mathbf{q}, \omega)$ $=\rho_{\alpha \alpha} /\left(1-i \omega / \Gamma_{0}+b q^{2}\right)$ where $\rho_{\alpha \alpha}=\chi_{\alpha \alpha}^{\prime}(\mathbf{Q}, 0)$ and $\Gamma_{0}$ is the relaxation rate of order $\left|y_{F}\right|=T_{K}$. As expected for the antiferromagnetic case, the relaxation rate remains finite when $q$ goes to zero reflecting the fact that the fluctuations of the order parameter are not conserved. The bare interband susceptibility $\bar{\chi}_{\alpha \beta}(\mathbf{Q}+\mathbf{q}, \omega)$ can be considered as purely real and frequency independent equal to $\rho_{\alpha \beta}$ in the lowfrequency limit. Due to the presence of the hybridization gap in the density-of-states, the continuum of the interband particle-hole excitations displays a gap ranging from $2 \sigma_{0}$, the value of the direct gap for the wave vector $\mathbf{0}$, to $2\left|y_{F}\right|$, the value of the indirect gap at $\mathbf{Q}$. The RPA dynamical spin susceptibility is given by

$$
\chi_{f f}^{R P A^{\prime \prime}}(\mathbf{Q}+\mathbf{q}, \omega)=\omega \frac{\chi_{f f}^{\prime}(\mathbf{Q}+\mathbf{q}) \Gamma(q)}{\omega^{2}+\Gamma(q)^{2}}
$$

with

$$
\begin{gathered}
\chi_{f f}^{\prime}(\mathbf{Q}+\mathbf{q})=\frac{\rho_{\alpha \alpha}+\rho_{\alpha \beta}}{\left(1-I_{q}\right)}, \\
\Gamma(q)=\Gamma_{0}\left(1-I_{q}\right),
\end{gathered}
$$

where $I_{q}=I-b q^{2}$ and $I=J_{S}^{2} \rho_{\alpha \alpha} \rho_{\alpha \beta}$ (of order 1 near the antiferromagnetic instability).

The bulk staggered susceptibility $\chi_{f f}^{\prime}(\mathbf{Q})$ diverges at $I$ $=1$. The frequency dependence of $\chi_{f f}^{R P A^{\prime \prime}}(\mathbf{Q}+\mathbf{q}, \boldsymbol{\omega})$ is a Lorentzian with a vanishing relaxation rate $\Gamma(0)$ at the antiferromagnetic transition. The corresponding excitation can be analyzed as an antiferromagnetic paramagnon mode that softens at the magnetic transition. Equation (12) provides us with the dispersion of that mode. The corresponding dynamical exponent $z$ is found to be equal to 2 due to the overdamping of the mode when enters the continuum of the $\alpha$ - $\alpha$ particle-hole excitations. We will show how the dynamic exponent $z$ strongly affects the static critical behavior. This is due to the fact that, contrary to the $T>0$ phase transitions, statics and dynamics are intimately linked in quantum ( $T$ $=0)$ phase transitions. We turn now to the resulting quantum-classical crossover occurring at finite temperature. 


\section{CRITICAL PHENOMENA AROUND \\ THE ANTIFERROMAGNETIC QUANTUM CRITICAL POINT (AF-QCP)}

The parameter which controls the temperature dependence of the thermodynamical variables near the AF-QCP is $\sigma_{f}^{2}$, which expresses the thermal local fluctuations of the staggered magnetization. Using Eqs. (11) and (12)

$$
\sigma_{f}^{2}=\sum_{\mathbf{q}} S(\mathbf{Q}+\mathbf{q}) \sim \int S(\mathbf{Q}+\mathbf{q}) q^{d-1} d q,
$$

where $S(\mathbf{Q}+\mathbf{q})$ is the static form factor

$$
S(\mathbf{Q}+\mathbf{q})=\frac{3}{\pi} \int_{0}^{\infty} \operatorname{coth}(\beta \omega / 2) \chi_{f f}^{\prime \prime}(\mathbf{Q}+\mathbf{q}, \omega) d \omega .
$$

Depending on the temperature scale considered, quantum or classical behavior is observed. In general, the thermal fluctuations $\sigma_{f}^{2}$ of the magnetization should depend on the total self-consistent renormalized dynamical spin susceptibility $\chi_{f f}^{\prime \prime}(\mathbf{Q}+\mathbf{q}, \omega)$ as defined in Eq. (9). However, in practice, we will be content with its truncated form $\chi_{f f}^{R P A^{\prime \prime}}(\mathbf{Q}+\mathbf{q}, \omega)$ derived at the RPA level. The temperature dependence of the static form factor $S(\mathbf{Q}+\mathbf{q})$ is given by

$$
S(\mathbf{Q}+\mathbf{q})=\frac{6 T}{\pi} \chi_{f f}^{\prime}(\mathbf{Q}+\mathbf{q}) \tan ^{-1}\left(\frac{T}{\Gamma(q)}\right) .
$$

A first crossover temperature $T_{I}=2 \Gamma_{0}(1-I)$ appears, which separates the quantum from the classical regime.

(i) $T<T_{I}$ : quantum regime. One can show that the static form factor $S(\mathbf{Q}+\mathbf{q})$ exhibits a quadratic temperature dependence

$$
S(\mathbf{Q}+\mathbf{q})=\frac{6}{\pi} \frac{\chi_{f f}^{\prime}(\mathbf{Q}+\mathbf{q})}{\Gamma_{0}\left(1-I_{q}\right)} T^{2},
$$

The thermal local fluctuations $\boldsymbol{\sigma}_{f}^{2}$ of the magnetization are also quadratic as a function of the temperature. They can be easily evaluated from Eq. (15). Taking advantage of the fact that the integrand $S(\mathbf{Q}+\mathbf{q}) q^{d-1}$ is peaked at $q_{1}$ $=\sqrt{(1-I) / b}$, the temperature dependence of $\boldsymbol{\sigma}_{f}^{2}$ is also found to be quadratic

$$
\boldsymbol{\sigma}_{f}^{2}=\frac{3}{2 \pi} \frac{\chi_{f f}^{\prime}(\mathbf{Q})}{\Gamma_{0}(1-I)} T^{2},
$$

The physics is quantum in the sense that the fluctuations on the scale of the magnetic correlation length $\xi$ have energy much greater than the temperature $k_{B} T$.

(ii) $T>T_{I}$ : classical regime. In this regime, the static form factor $S(\mathbf{Q}+\mathbf{q})$ shows a linear temperature dependence. As long as $q$ is smaller than the thermal cutoff $q^{*}$ defined by $\omega_{\max }\left(q^{*}\right)=T, S(\mathbf{Q}+\mathbf{q})$ takes the following form

$$
S(\mathbf{Q}+\mathbf{q})=3 \chi_{f f}^{\prime}(\mathbf{Q}+\mathbf{q}) T .
$$

The thermal local fluctuations $\sigma_{f}^{2}$ of the magnetization are deduced from Eq. (15). The main contribution arises from the integration over $\left[q_{1}, q^{*}\right]$. The result depends on the dimensionality $d$. One finds $\sigma_{f}{ }^{2} \sim T^{3 / 2}$ at $d=3$ and $\sigma_{f}^{2}$
TABLE I. Predictions for thermal local fluctuations $\boldsymbol{\sigma}_{f}^{2}$ of the staggered magnetization depending on the temperature $T$ and on the dimensionality $d$. $T_{I} \sim(1-I)$ represents the first crossover temperature separating the quantum from the classical regime in the vicinity of the antiferromagnetic-quantum critical point (AF-QCP).

\begin{tabular}{ccc}
\hline \hline & Quantum regime $T<T_{I}$ & Classical regime $T>T_{I}$ \\
\hline$d=3$ & $\sigma_{f}^{2} \sim \chi_{f f}^{\prime}(Q) T^{2} / \Gamma_{0}$ & $\sigma_{f}^{2} \sim T^{3 / 2}$ \\
$d=2$ & $\sigma_{f}^{2} \sim \chi_{f f}^{\prime}(Q) T^{2} / \Gamma_{0}$ & $\sigma_{f}^{2} \sim T \ln T$ \\
\hline
\end{tabular}

$\sim T \ln T$ at $d=2$. This classical regime corresponds to the case where the energy of the mode on the scale of $\xi$ becomes less than $k_{B} T$.

Table I recapitulates the expressions that we get for the thermal local fluctuations $\sigma_{f}^{2}$ of the staggered magnetization depending on the temperature scale and on the dimensionality. Following Eq. (10), the staggered spin susceptibility is given by

$$
\chi_{Q}^{\prime}=\frac{\chi_{f f}^{0^{\prime}}(Q)}{1-I+\lambda \sigma_{f}^{2}}
$$

where $\chi_{f f}^{0^{\prime}}(Q)=\rho_{\alpha \alpha}+\rho_{\alpha \beta}$. Incorporating the values of the thermal local fluctuations $\boldsymbol{\sigma}_{f}^{2}$ reported in Table I, we are able to identify a second crossover temperature $T_{I I}$. Above $T I I, \quad \sigma_{f}^{2}$ becomes larger than $(1-I)$ and all the physical quantities are controlled by the temperature only. $T_{I I} \sim(1$ $-I)^{2 / 3}$ at $d=3$ and $T_{I I} \sim(1-I)$ at $d=2$. As expected in the two-dimensional case, the $T=0$ transition is at its upper critical dimension since $z+d=4$ in the antiferromagnetic case characterized by a dynamic exponent $z=2$. That corresponds to the marginal case for which the intermediate regime $T_{I}<T<T_{I I}$ is squeezed out of existence. The results for the $d=3$ and $d=2$ cases are, respectively, summarized in Figs. 1 and 2, which picture the different regimes of behaviors reached depending on the values of the temperature $T$ and of the control parameter $I=J^{2} \rho_{\alpha \alpha} \rho_{\alpha \beta}$. When $I>1$, long-range antiferromagnetic order occurs when $T$ is smaller than the Néel temperature $T_{N} \cdot T_{N} \sim(I-1)^{2 / 3}$ at $d=3$ and $T_{N} \sim(I-1)$ at $d=2$.

\section{DISCUSSION}

The low-temperature physics is dominated by the presence of an antiferromagnetic paramagnon mode that softens at the magnetic transition. The results depend crucially on the value of $d+z$, where $d$ is the spatial dimension and $z$ is the dynamic exponent associated to that mode. Due to the overdamping of the mode when it enters the continuum of intraband particle-hole excitations, the dynamic exponent $z$ is found to be equal to 2 in the antiferromagnetic case. As far as the dimensionality is concerned, there is no doubt about the dimensionality associated with the heavy quasiparticles in those systems that are clearly of 3 . However the question about the dimensionality of the critical magnetic fluctuations, which the quasiparticles are coupled to, is still an open problem. Up to now, it has been assumed that the critical fluctuations of the quantum phase transition (QPT) are dominated by the three-dimensional antiferromagnetic 


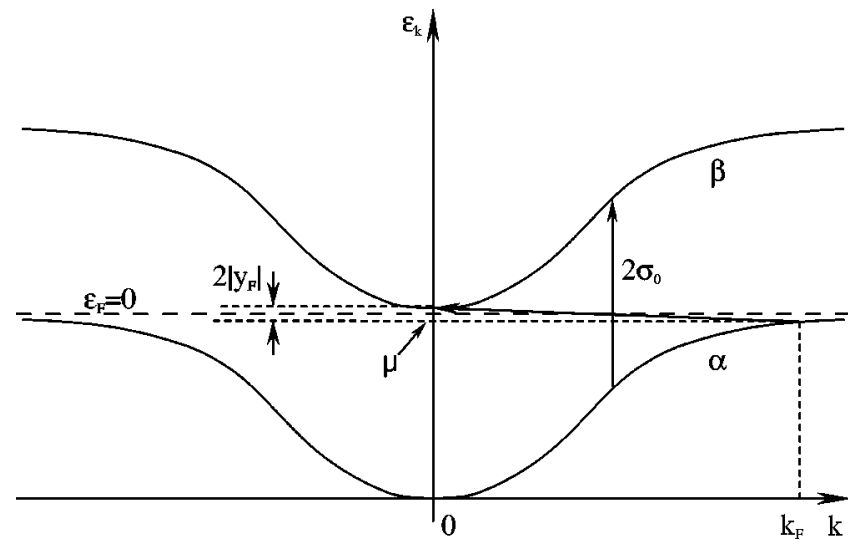

FIG. 1. Phase diagram in the plane $(T, I)$ for dimension equal to 3 . The shaded region represents the long-range antiferromagnetic phase bordered by the Néel temperature $T_{N}$. The unshaded region marks the magnetically-disordered regimes $I, I I$, and $I I I$, associated to different behaviors of the system. Regime $I$ is the quantum regime in which the energy of the relevant mode on the scale of $\xi$ is much greater than $k_{B} T$. The magnetic correlation length is $\xi$ $\sim 1 / \sqrt{(1-I)}$ and the staggered spin susceptibility is $\chi_{Q}^{\prime}$ $=\chi_{f f}^{0^{\prime}}(Q) /\left(1-I+a T^{2}\right)$. Regimes $I I$ and $I I I$ are both classical regimes in which the thermal effects are important since the fluctuations on the scale of $\xi$ have energy much smaller than $k_{B} T$. In Regime $I I, \xi \sim 1 / \sqrt{(1-I)}$ is still controlled by $(1-I)$ but the staggered spin susceptibility is sensitive to the thermal fluctuations: $\chi_{Q}^{\prime}=\chi_{f f}^{0^{\prime}}(Q) /\left(1-I+a T^{3 / 2}\right)$. In Regime $I I I$, both $\xi$ and $\chi_{Q}^{\prime}$ are controlled by the temperature: $\xi \sim 1 / T^{3 / 4}$ and $\chi_{Q}^{\prime}=\chi_{f f}^{0^{\prime}}(Q) / T^{3 / 2}$.

correlations. This would impose a description by a quantum critical theory with $d=3, z=2$. Recently Rosch et al. ${ }^{28}$ proposed, on the basis of the neutron-scattering data in $\mathrm{CeCu}_{6-x} \mathrm{Au}_{x}$, that the critical magnetic fluctuations are two dimensional, which leads to a QCP with $d=2$ and $z=2$. Let us summarize the different regimes of behaviors that we get in each of those two cases $(z=2, d=3$ or 2) depending on the values of the temperature $T$ and of the control parameter $I=J^{2} \rho_{\alpha \alpha} \rho_{\alpha \beta}$. As one can see, the results that we obtain are very similar to those established by Hertz $^{20}$ and Millis ${ }^{11}$ us-
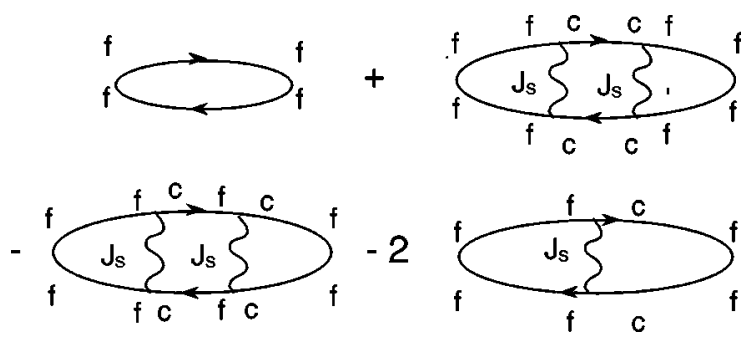

FIG. 2. Phase diagram in the plane $(T, I)$ for dimension equal to 2 . The shaded region represents the long-range antiferromagnetic phase bordered by the Néel temperature $T_{N}$. The unshaded region marks the magnetically-disordered regimes $I$ and $I I I$. In that $d=2$ case, the equivalent of regime $I I$ in Fig. 1 is squeezed out of existence since $T_{I}=T_{I I}$. Regime $I$ is the quantum regime in which the thermal fluctuations are negligible: $\xi \sim 1 / \sqrt{(1-I)}$ and $\chi_{Q}^{\prime}$ $=\chi_{f f}^{0^{\prime}}(Q) /\left(1-I+a T^{2}\right)$. Regime $I I I$ is the unique classical regime in which both the magnetic correlation length and the staggered spin susceptibility are controlled by the temperature: $\xi \sim 1 / \sqrt{T \ln T}$ and $\chi_{Q}^{\prime}=\chi_{f f}^{0^{\prime}}(Q) /(T \ln T)$. ing renormalization-group approaches in the spin-fluctuation theory.

(i) Case $d=3$. A long-range antiferromagnetic phase occurs when $I>1$ below the Néel temperature $T_{N} \sim(I-1)^{2 / 3}$. A first crossover temperature $T_{I} \sim(1-I)$ separates the quantum from the classical regime. In the regime $I(I<1)$ and $T$ $<T_{I}$ ), the physics is quantum in the sense that the energy of the relevant mode on the scale of $\xi$ is greater than the temperature $k_{B} T$. Since $d+z>4$, the $T=0$ phase transition is above its upper critical dimension and the various physical quantities depend upon the parameter $I$. The magnetic correlation length diverges at the magnetic transition $\xi$ $\sim 1 / \sqrt{(1-I)}$ and the staggered spin susceptibility behaves as $\chi_{Q}^{\prime}=\chi_{f f}^{0^{\prime}}(Q) /\left(1-I+a T^{2}\right)$. Regimes $I I$ and $I I I$ are both classical regimes characterized by large thermal effects since the fluctuations on the scale of $\xi$ have energy much smaller than $k_{B} T$. In regime $I I\left[T_{I}<T<T_{I I}\right.$ with $\left.T_{I I} \sim(1-I)^{2 / 3}\right]$, the magnetic correlation length $\xi \sim 1 / \sqrt{(1-I)}$ is still controlled by $(1-I)$ even though modes at the scale of $\xi$ have energies less than $k_{B} T$. On the contrary, the staggered spin susceptibility is sensitive to the thermal fluctuations $\chi_{Q}^{\prime}$ $=\chi_{f f}^{0^{\prime}}(Q) /\left(1-I+a T^{3 / 2}\right)$. In regime $I I I\left(T>T_{I I}\right)$, the thermal dependence of physical quantities becomes universal and both $\xi$ and $\chi_{Q}^{\prime}$ are controlled by the temperature: $\xi \sim 1 / T^{3 / 4}$ and $\chi_{Q}^{\prime}=\chi_{f f}^{0^{\prime}}(Q) / T^{3 / 2}$.

(ii) Case $d=2$. Then, since $z=2, z+d=4$ and the $T$ $=0$ phase transition is at its upper critical dimension. The physics is qualitatively similar to the case $d=3$ with stronger fluctuation effects particularly in the classical regime. A long-range antiferromagnetic phase occurs when $I>1$ below the Néel temperature $T_{N} \sim(I-1)$. For $d=2$, the two crossover temperatures $T_{I}$ and $T_{I I}$ coincide so that regime $I I$ of Fig. 1 is squeezed out of existence. Regime $I\left[T<T_{I}\right.$ with $\left.T_{I} \sim(1-I)\right]$ is the quantum regime in which the thermal fluctuations are negligible : $\xi \sim 1 / \sqrt{(1-I)}$ and $\chi_{Q}^{\prime}$ $=\chi_{f f}^{0^{\prime}}(Q) /\left(1-I+a T^{2}\right)$. Regime $I I I\left(T>T_{I}\right)$ is the unique classical regime characterized by very strong fluctuation effects since $k_{B} T$ is larger than the energy of the relevant mode on the scale of $\xi$. Both the magnetic correlation length and the staggered spin susceptibility are then controlled by the temperature: $\xi \sim 1 / \sqrt{T \ln T}$ and $\chi_{Q}^{\prime}=\chi_{f f}^{0^{\prime}}(Q) /(T \ln T)$.

\section{CONCLUDING REMARKS}

We considered the $S=1 / 2$ Kondo lattice model in a selfconsistent one-loop approximation starting from a generalized Hubbard-Stratonovich transformation of the Kondo interaction term. The model exhibits a zero-temperature magnetic phase transition at a critical value of the Kondo coupling. The transition is usually antiferromagnetic but it may be incommensurate depending on the band structure considered. The low-temperature physics is controlled by a collective mode that softens at the antiferromagnetic transition with a dynamic exponent $z$ equal to 2 .

A quantum-classical crossover occurs at a temperature $T_{I}$ related to the characteristic energy scale of that mode. Heavy-fermion systems are usually believed to be threedimensional. However, since some recent inelastic-neutronscattering experiments performed in $\mathrm{CeCu}_{6-x} \mathrm{Au}_{x}$ show that 
the critical magnetic fluctuations, which the quasiparticles are coupled to, are two-dimensional, we both considered the cases $d=2$ and $d=3$ with $z=2$. The low-temperature behavior of the system is deduced, with predictions for the temperature dependence of the physical quantities such as the magnetic correlation length $\xi$ and the staggered susceptibility $\chi_{Q}^{\prime}$. A second crossover temperature $T_{I I}$ appears above which the thermal dependence of $\xi$ and $\chi_{Q}^{\prime}$ becomes universal and is uniquely controlled by the temperature. It would be very interesting now to study the temperature behavior of other physical quantities as the specific heat or the transport properties.

A number of significant issues remain to be addressed. Among others, we will mention the possibility of getting a different value of the dynamic exponent for instance $z=1$ if nesting effects are considered corresponding to an absence of damping of the mode when located in the gap of excitations around the antiferromagnetic vector. Disorder is expected to play a crucial role in this problem. In that direction, some recent works ${ }^{33,17}$ pointed out the formation of some "hot lines," i.e., points at the Fermi surface linked by the magnetic $\mathbf{Q}$ vector. On the hot lines, the quasiparticle scattering rate is linear in temperature while, away from the hot lines, it acquires the standard Fermi liquid form in $T^{2}$. At low temperature, the "cold" regions are shown to short circuit the scattering near those lines, and induce a $T^{2}$ behavior of the resistivity. The disorder is found to emphasize the contribution of the hot lines, eventually leading to a non-Fermi liquid behavior. It would be worthy to study the effects of the "hot lines" in the presence of disorder within the critical phenomena description presented in this paper.

\section{ACKNOWLEDGMENTS}

We would like to thank Nick Bernhoeft, Piers Coleman, Mucio Continentino, Jacques Flouquet, Patrick A. Lee, and Gilbert Lonzarich for very useful discussions on this work.

\section{APPENDIX EXPRESSIONS OF THE DIFFERENT BUBBLES}

The expressions of the different bubbles appearing in the expression of the boson propagators [cf. Eq. (5)] are given here (with $\mathrm{i}=1,2, \mathrm{~m}$, or ff)

$$
\begin{array}{r}
\bar{\varphi}_{i}\left(\mathbf{q}, i \omega_{\nu}\right)=\varphi_{i}\left(\mathbf{q}, i \omega_{\nu}\right)+\varphi_{i}\left(-\mathbf{q},-i \omega_{\nu}\right), \\
\varphi_{1}\left(\mathbf{q}, i \omega_{\nu}\right)=-\frac{1}{\beta} \sum_{k \sigma, i \omega_{n}} G_{c f_{0}}^{\sigma}\left(\mathbf{k}+\mathbf{q}, i \omega_{n}+i \omega_{\nu}\right) G_{f f_{0}}^{\sigma}\left(\mathbf{k}, i \omega_{n}\right), \\
\varphi_{2}\left(\mathbf{q}, i \omega_{\nu}\right)=-\frac{1}{\beta} \sum_{k \sigma, i \omega_{n}} G_{c c_{0}}^{\sigma}\left(\mathbf{k}+\mathbf{q}, i \omega_{n}+i \omega_{\nu}\right) G_{f f_{0}}^{\sigma}\left(\mathbf{k}, i \omega_{n}\right), \\
\varphi_{m}\left(\mathbf{q}, i \omega_{\nu}\right)=-\frac{1}{\beta} \sum_{k \sigma, i \omega_{n}} G_{c f_{0}}^{\sigma}\left(\mathbf{k}+\mathbf{q}, i \omega_{n}+i \omega_{\nu}\right) G_{c f_{0}}^{\sigma}\left(\mathbf{k}, i \omega_{n}\right), \\
\varphi_{f f}^{\|}\left(\mathbf{q}, i \omega_{\nu}\right)=-\frac{1}{\beta} \sum_{k \sigma, i \omega_{n}} G_{f f_{0}}^{\sigma}\left(\mathbf{k}+\mathbf{q}, i \omega_{n}+i \omega_{\nu}\right) G_{f f_{0}}^{\sigma}\left(\mathbf{k}, i \omega_{n}\right),
\end{array}
$$

$$
\begin{aligned}
G_{c c}\left(\mathbf{k}, i \omega_{n}\right) & =v_{k}^{2} G_{\alpha \alpha}\left(\mathbf{k}, i \omega_{n}\right)+u_{k}^{2} G_{\beta \beta}\left(\mathbf{k}, i \omega_{n}\right), \\
G_{c f}\left(\mathbf{k}, i \omega_{n}\right) & =G_{f c}\left(\mathbf{k}, i \omega_{n}\right) \\
& =-u_{k} v_{k}\left[G_{\alpha \alpha}\left(\mathbf{k}, i \omega_{n}\right)-G_{\beta \beta}\left(\mathbf{k}, i \omega_{n}\right)\right],
\end{aligned}
$$

where $G_{\alpha \alpha}\left(\mathbf{k}, i \omega_{n}\right)$ and $G_{\beta \beta}\left(\mathbf{k}, i \omega_{n}\right)$ are the Green's functions associated to the eigenstates $\alpha_{k \sigma}^{\dagger}|0\rangle$ and $\beta_{k \sigma}^{\dagger}|0\rangle$. In the low-frequency limit, one can easily check that the dynamical spin susceptibility may be written as

$$
\chi_{f f}\left(\mathbf{q}, i \omega_{\nu}\right)=\frac{\chi_{\alpha \alpha}\left(\mathbf{q}, i \omega_{\nu}\right)+\bar{\chi}_{\alpha \beta}\left(\mathbf{q}, i \omega_{\nu}\right)}{1-J_{S}^{2} \chi_{\alpha \alpha}\left(\mathbf{q}, i \omega_{\nu}\right) \bar{\chi}_{\alpha \beta}\left(\mathbf{q}, i \omega_{\nu}\right)},
$$

for both the longitudinal and the transverse parts.

$$
\begin{aligned}
\chi_{\alpha \alpha}\left(\mathbf{q}, i \omega_{\nu}\right)= & \frac{1}{\beta} \sum_{k} \frac{n_{F}\left(E_{k}^{-}\right)-n_{F}\left(E_{k+q}^{-}\right)}{i \omega_{\nu}-E_{k+q}^{-}+E_{k}^{-}} \\
\bar{\chi}_{\alpha \beta}\left(\mathbf{q}, i \omega_{\nu}\right)= & \frac{1}{\beta} \sum_{k}\left(u_{k}^{2} v_{k+q}^{2}+v_{k}^{2} u_{k+q}^{2}\right) \\
& \times \frac{n_{F}\left(E_{k}^{-}\right)-n_{F}\left(E_{k+q}^{+}\right)}{i \omega_{\nu}-E_{k+q}^{+}+E_{k}^{-}} .
\end{aligned}
$$


*Also at the Center National de la Recherche Scientifique (CNRS).

${ }^{1}$ For reviews see H. von Lohneysen, J. Phys.: Condens. Matter 8, 9689 (1996); D. A. Gajewski, N. R. Dilley, R. Chau, and M. B. Maple, ibid. 8, 9793 (1996); F. Steglich, B. Buschinger, P. Gegenwart, M. Lohmann, R. Helfrich, C. Langhammer, P. Hellmann, L. Donnevert, S. Thomas, A. Link, C. Geibel, M. Lang, G. Sparn, and W. Assmus, ibid. 8, 9909 (1996).

${ }^{2}$ H. von Löhneysen, A. Schröder, M. Sieck, and T. Trappmann, Phys. Rev. Lett. 72, 3262 (1994); H. von Löhneysen, J. Phys.: Condens. Matter 8, 9689 (1996); O. Stockert, H. V. Löhneysen, A. Schröder, M. Loewenhaupt, N. Pyka, P. L. Gammel, and U. Yaron, Physica B 230-232, 247 (1997).

${ }^{3}$ N. D. Mathur, F. M. Grosche, S. R. Julian, I. R. Walker, D. M. Freye, R. K. W. Haselwimmer, and G. G. Lonzarich, Nature (London) 394, 39 (1998), and references therein.

${ }^{4}$ F. Steglich, B. Buschinger, P. Gegenwart, M. Lohmann, R. Helfrich, C. Langhammer, P. Hellmann, L. Donnevert, S. Thomas, A. Link, C. Geiber, M. Lang, G. Sparn, and W. Assmus, J. Phys.: Condens. Matter 8, 9909 (1996).

${ }^{5}$ C. L. Seaman, M. B. Maple, B. W. Lee, S. Ghamaty, M. S. Torikachvili, J.-S. Kang, L.-Z. Liu, J. Allen, and D. L. Cox, Phys. Rev. Lett. 67, 2882 (1991).

${ }^{6}$ S. Kambe, S. Raymond, L. P. Regnault, J. Flouquet, P. Lejay, and P. Haen, J. Phys. Soc. Jpn. 65, 3294 (1996).

${ }^{7}$ E. Miranda, V. Dobrosavljevic, and G. Kotliar, J. Phys.: Condens. Matter 8, 9871 (1996).

${ }^{8}$ A. H. Castro-Neto, G. Castilla, and B. A. Jones, Phys. Rev. Lett. 81, 3531 (1998); M. C. de Andrade, R. Chau, R. P. Dickey, N. R. Dilley, E. J. Freeman, D. A. Gajewski, M. B. Maple, R. Movshovich, A. H. Castro Neto, G. Castilla, and B. A. Jones, ibid. 81, 5620 (1998).

${ }^{9}$ R. Narayanan, T. Vojta, D. Belitz, T. R. Kirkpatrick, Phys. Rev. B 60, 10150 (1999); T. R. Kirkpatrick and D. Belitz, Phys. Rev. Lett. 76, 2571 (1996).

${ }^{10}$ B. Andraka and A. M. Tsvelik, Phys. Rev. Lett. 67, 2886 (1991).

${ }^{11}$ A. J. Millis, Phys. Rev. B 48, 7183 (1993).
${ }^{12}$ T. Moriya and T. Takimoto, J. Phys. Soc. Jpn. 64, 960 (1995).

${ }^{13}$ M. A. Continentino, Phys. Rev. B 47, R11 587 (1993).

${ }^{14}$ P. Schlottmann, Phys. Rev. B 59, 12379 (1999).

${ }^{15}$ P. Coleman, Physica B 259-261, 353 (1999).

${ }^{16}$ Q. Si, J. L. Smith, and K. Ingersent, Int. J. Mod. Phys. B 13, 2331 (1999).

${ }^{17}$ A. Rosch, Phys. Rev. Lett. 82, 4280 (1999).

${ }^{18}$ A. Schröder, G. Aeppli, E. Bucher, R. Ramazashvili, and P. Coleman, Phys. Rev. Lett. 80, 5623 (1998).

${ }^{19}$ D. L. Cox, Phys. Rev. Lett. 59, 1240 (1987); D. L. Cox and M. Jarrell, J. Phys.: Condens. Matter 8, 9825 (1996).

${ }^{20}$ J. A. Hertz, Phys. Rev. B 14, 1165 (1976).

${ }^{21}$ T. Moriya and A. Kawabata, J. Phys. Soc. Jpn. 34, 639 (1973); H. Hasegawa and T. Moriya, ibid. 36, 1542 (1974).

${ }^{22}$ S. Doniach, Physica B 91, 231 (1977); C. Lacroix and M. Cyrot, Phys. Rev. B 20, 1969 (1979); D. M. Newns and N. Read, Adv. Phys. 36, 799 (1987); and for a recent review see H. Tsunetsugu, M. Sigrist, and K. Ueda, Rev. Mod. Phys. 69, 809 (1997) and references therein.

${ }^{23}$ A. J. Millis and P. A. Lee, Phys. Rev. B 35, 3394 (1987); A. Auerbach and K. Levin, Phys. Rev. Lett. 57, 877 (1986); M. Lavagna, A. J. Millis, and P. A. Lee, ibid. 58, 266 (1987).

${ }^{24}$ A. Houghton, N. Read, and H. Won, Phys. Rev. B 37, 3782 (1988).

${ }^{25}$ C. Pépin and M. Lavagna, Phys. Rev. B 59, 2591 (1999).

${ }^{26} \mathrm{P}$. Nozières (unpublished).

${ }^{27}$ J. A. Hertz and M. A. Klenin, Phys. Rev. B 10, 1084 (1974); J. A. Hertz and M. A. Klenin, Physica B 91, 49 (1977).

${ }^{28}$ A. Rosch, A. Schröder, O. Stockert, and H. von Löhneysen, Phys. Rev. Lett. 79, 159 (1997).

${ }^{29}$ N. Read and D. N. Newns, J. Phys. C 16, 3273 (1983).

${ }^{30}$ G. G. Lonzarich and L. Taillefer, J. Phys. C 18, 4339 (1985).

${ }^{31}$ N. R. Bernhoeft and G. G. Lonzarich, J. Phys.: Condens. Matter 7, 7325 (1995).

${ }^{32}$ Y. Kuramoto and K. Miyake, J. Phys. Soc. Jpn. 59, 2831 (1990).

${ }^{33}$ R. Hlubina and T. M. Rice, Phys. Rev. B 51, 9253 (1995). 